\title{
Cellular immune activation in Sardinian middle-aged, older adults and centenarians
}

\author{
Salvatore Sotgia ${ }^{\mathrm{a}, *}$, Angelo Zinellu ${ }^{\mathrm{a}}$, Arduino A. Mangoni ${ }^{\mathrm{b}}$, Roberta Serra ${ }^{\mathrm{c}}$, Gianfranco Pintus ${ }^{\mathrm{d}}$, \\ Calogero Caruso ${ }^{\mathrm{e}}$, Luca Deiana ${ }^{\mathrm{a}}$, Ciriaco Carru ${ }^{\mathrm{a}, \mathrm{f}}$ \\ ${ }^{a}$ Department of Biomedical Sciences, School of Medicine, University of Sassari, Sassari, Italy \\ b Department of Clinical Pharmacology, School of Medicine, Flinders University and Flinders Medical Centre, Adelaide, Australia \\ c University Hospital of Sassari (AOU-SS), Sassari, Italy \\ d Department of Biomedical Sciences, College of Health Sciences, Qatar University, Doha, Qatar \\ e Department of Biopathology and Biomedical Methodology, University of Palermo, Palermo, Italy \\ f Quality Control Unit, University Hospital of Sassari (AOU-SS), Sassari, Italy
}

\section{A R T I C L E I N F O}

Section Editor: Richard Aspinall

Keywords:

Aging

Immunosenescence

Inflammaging

Longevity

Centenarians

Cytokines

Pteridines

\begin{abstract}
A B S T R A C T
In addition to viral infections, malignant disorders, autoimmune diseases, and allograft rejection episodes, neopterin increases in older people where it is found to be predictive of overall mortality. Thus, the serum concentrations of this biomarker of systemic immune and inflammation activation, were measured in a small cohort of Sardinian middle-aged, older adults and centenarians. There was a significant positive correlation between neopterin concentrations and age with the subjects in the 95-year-old group with the highest values. Notably, the group of centenarians had neopterin values comparable to those of 80- and 90-year-old groups, and significantly lower than that of 95-year-old group. This suggests a decreased monocyte/macrophage-mediated immune activation and an apparently preserved immune status in centenarians.
\end{abstract}

\section{Introduction}

The alteration in the immune response with advancing age, i.e., the immunosenescence (IS), has been well documented in humans and contributes to the increased morbidity and mortality in the older population (Ginaldi et al., 2001). IS may lead, in fact, to a decrease in the immune competence resulting in an increased susceptibility to infectious diseases and a poor response to immunization (Wu and Meydani, 2008). Aside from immunodeficiency, old age is also characterized by a greater susceptibility to autoimmune responses and by a persistent systemic inflammatory state (Oishi and Manabe, 2016) termed inflammaging (IF) (Franceschi and Campisi, 2014). The latter is characterized by elevated concentrations of proinflammatory cytokines, clotting factors, and acute phase reactants (Shaw et al., 2013). Although IF is usually subclinical and of low grade, it leads to long-term tissue degeneration involved in the pathogenesis of several non-communicable metabolic (Oishi and Manabe, 2016), neurodegenerative (Licastro et al., 2003), neoplastic (Oishi and Manabe, 2016), cardiovascular (Ridker et al., 2000), and autoimmune (Capuron et al., 2014) age-related diseases. The chronic activation of the immune system, proposed as a likely causal agent in such process, appears to be linked to the evolutionary, unpredicted, antigenic load caused by clinical and subclinical infections as well as the exposure to noninfective antigens (Baylis et al., 2013). Persistent exposure to antigens causes, in fact, a lifelong, chronic antigenic stress leading to adaptive processes resulting in an unspecific activation of macrophages and immunity cells that exhibit a senescent phenotype (Straub, 2015). The continuous proinflammatory load would induce a chronic immune stimulation, with an overproduction of activated cells and an overexpression of apoptosislinked receptors in lymphocytes (Straub, 2015). The senescent cells, in turn, would become less prone to spontaneous apoptosis, thus filling the immunological space in peripheral lymphoid organs (Straub, 2015). This results in a state of impaired immune response and concomitant upregulation of the inflammatory response (Fulop et al., 2016). Older adults in the 'old-old' subgroup, particularly centenarians, appear to escape from the effects of immune dysregulation and IF, with a resulting delay in disease onset. The identification of measurable circulating biomarkers of immune activation status in older adults might be useful to identify specific genotypic and/or phenotypic patterns associated with healthy aging, and to enhance disease risk stratification. Neopterin is a pyrazino-pyrimidine compound synthesized by the principal cell population of the innate immune system (macrophages)

\footnotetext{
* Corresponding author at: Department of Biomedical Sciences, University of Sassari, Viale San Pietro 43/B -I-07100 SASSARI, Italy

E-mail address: ssotgia@uniss.it (S. Sotgia).
} 
in response to one of the principal cytokines regulating adaptive immune response (interferon- $\gamma$, IFN- $\gamma$ ), produced by activated type 1 helper T-cells (Th1) (Hamerlinck, 1999; Melichar et al., 2006). Neopterin concentrations in human biological fluids are a sensitive marker of systemic immune and inflammatory activation (Fuchs et al., 1992; Murr et al., 2002), and have been shown to increase during viral infections, malignant disorders, autoimmune diseases, and allograft rejection episodes (Murr et al., 2009). Moreover, as neopterin production is also associated with increased production of reactive oxygen species and with low serum concentrations of antioxidants such as alpha-tocopherol (Murr et al., 2009), it can be also used as a marker of reactive oxygen species formed by the activated cellular immune system (Murr et al., 1999). With this background in mind, we aimed to investigate the immune activation status in different age groups within a Sardinian older population that included centenarians, by measuring the plasma concentrations of neopterin. We tested the hypothesis that the concentrations of neopterin are relatively lower in centenarians, reflecting a decreased cellular immune activation in this age group.

\section{Material and methods}

\subsection{Study population}

The participants were recruited within the framework of a wider research project for the discovery of molecular and genetic/epigenetic signatures underlying resistance to age-related diseases and comorbidities. Eligible participants were randomly selected from the electoral roll within the provinces of Sardinia region (Italy) and contacted by telephone by a trained study recruitment officer. Criteria for inclusion in the study were age, born and living in Sardinia, not being hospitalized, living at home (alone or with family), and the absence of acute illnesses. Thus, a total of 149 subjects, 48 males, and 101 females, aged 60 to 104 years and residing in different municipalities of the Sardinia region, were enrolled according to the characteristic male-tofemale sex ratio around 1:2.7 observed among Sardinian centenarians (Passarino et al., 2002). The study sample was then grouped into 5 age categories: 60-year-old (mean age (range) $60.7(60-62)$ years, $n=31$ ), 80 -year-old (mean age (range) $81.3(80-89)$ years, $n=32$ ), 90-year-old (mean age (range) 91.2 (90-94) years, $n=37$ ), 95-year-old (mean age (range) 95.9 (95-99) years, $n=23$ ), and 100-year-old (mean age (range) 100.9 (100-104) years, $n=27)$. An interview questionnaire was used to collect detailed information about existing chronic medical conditions and life-style habits of the participants. The interview was conducted after the blood sampling, and the questionnaire was filled out by the observer based on the answers given by the participants. All subjects were able to answer without the help of third-parties. Ethics approval to conduct the study was granted by the University of Sassari. The study was performed in accordance with the guidelines of the Declaration of Helsinki. All participants gave written informed consent before entering in the study.

\subsection{Blood sample collection and biochemical analyses}

Fasting blood samples were obtained in the morning after an approximately ten-hour overnight and anyway within 9:30 am. Samples were collected by venipuncture into EDTA-treated plain tubes or tubes without anticoagulant and centrifuged at $4{ }^{\circ} \mathrm{C}$ and $3000 \times g$ for $10 \mathrm{~min}$ to separate, respectively, plasma or serum, which were then stored at $-80^{\circ} \mathrm{C}$ until analysis. Without previous freeze-thaw cycles, neopterin was measured by an ultra-performance liquid chromatographer (UPLC) with fluorescence detection as described by Carru et al. (2004) with minor adjustments. Briefly, $50 \mu \mathrm{L}$ of a $15 \%(w / v)$ trichloroacetic acid solution were added to $100 \mu \mathrm{L}$ of a standard or plasma sample to precipitate the proteins, and centrifuged at $10,600 \times g$ for $10 \mathrm{~min}$ at $4{ }^{\circ} \mathrm{C}$. A $20 \mu \mathrm{L}$-volume of clear supernatant was injected into UPLC and the ultra-performance liquid chromatography was carried out at room temperature on a $200 \mathrm{~mm} \times 5 \mathrm{~mm}$ reversed-phase Waters Spherisorb ODS $/ 25 \mu \mathrm{m}$ column at a flow rate of $1.2 \mathrm{~mL} \mathrm{~min}^{-1}$ with water:acetonitrile (98:2) as the mobile phase. Neopterin was detected making use of its natural fluorescence at an excitation wavelength of $353 \mathrm{~nm}$ and an emission wavelength of $438 \mathrm{~nm}$. Plasma creatinine and glutathione (GSH) were assayed as described by Zinellu et al. (2003, 2004) as indexes of renal function and of the oxidative stress, respectively. Serum folate and vitamin B12 were measured by using an IMX Analyzer (Abbott Labs, Abbott Park, IL, USA) as indexes of nutritional status (Green, 2011).

\subsection{Statistical}

Data were checked for normality of distribution using the Kolmogorov-Smirnov test and were presented either as mean \pm SD or median and interquartile range (IQR) if data distribution was skewed. Depending on the normality of the distribution of the variables, associations between continuous variables were assessed using Pearson or Spearman rank correlation coefficients as appropriate. Differences between groups were assessed by ANOVA or Kruskal-Wallis test as well as by independent $t$-test or Mann-Whitney test (independent samples) as appropriate. When a significant difference was observed, the pairwise comparison of subgroups was performed by a Student-Newman-Keuls test for ANOVA or according to Conover (1999) for the Kruskal-Wallis test. Jonckheere-Terpstra trend test was performed to check if medians were ordered (increase or decrease) according to the order of the qualitative factor. Partial correlation analysis after $\log 10$ transformation of the non-normally distributed variables was used to assess the contribution of different variables to plasma neopterin concentrations. Chi-squared test was used to test for significant differences in the frequency of clinical status between age groups. A two-sided $P$ value of 0.05 was chosen as the cut-off for statistical significance. Statistical analyses were performed using MedCalc Statistical Software for Windows, version 17.5.5 64 bit (MedCalc Software bvba, Ostend, Belgium).

\section{Results}

Subjects in the 60-year-old group had no known history of chronic diseases whereas about $60 \%$ of participants in the other groups reported one or more medical conditions such as hypertension $(78.8 \%)$, diabetes $(12.2 \%)$, hypercholesterolemia $(10.1 \%)$, asthma $(7.1 \%)$, and hearth failure (4.1\%). The prevalence of chronic disease in centenarians was lower than other groups, although this difference was not statistically significant (66\% vs. $75 \%$, chi-squared $=0.0117, P=0.91$ ). Most of the subjects were either non-smokers or former smokers, and only $2.9 \%$ were current smokers. Non-drinkers, former drinkers and current drinkers represented the $25.7 \%, 42.9 \%$, and $31.4 \%$ of the study population, respectively. Table 1 outlines the biochemical parameters considered in the study population. The distribution of plasma neopterin concentration was skewed, with slightly more than half of the participants (52\%) having values ranging between 10 and $30 \mathrm{nM}$. Median neopterin concentration was 20.35 (IQR 11.7-32.2) nM, and there were no significant gender-related differences (females 19.09 (IQR 11.2-30.7) nM vs. males 22.4 (IQR 13.1-33.1) nM, $P=0.54$ ). There was a significant positive correlation between neopterin concentrations and age (Spearman rank correlation coefficient $=0.27 ; P=0.0008$ ). Partial correlation analysis by using gender and the concentrations of creatinine, folate, glutathione and vitamin B12 as covariates, strengthen the independent association between age and plasma neopterin concentrations $(P=0.0026)$. However, as displayed in Fig. 1, which shows a local regression smoothing trendline, a trend towards lower neopterin concentrations was observed with age above $\sim 92$ years. As displayed in Fig. 2, neopterin concentrations significantly differed among the five age groups $(P=0.0002)$. Post-hoc analysis for pairwise comparisons shows a significant age-associated increase in neopterin concentrations up to the 95-year-old group 
Table 1

Biochemical characteristics of the 149 subjects.

\begin{tabular}{|c|c|c|c|c|c|c|}
\hline & All $(n=149)$ & 60 -Year-old $(n=30)$ & 80 -Year-old $(n=32)$ & 90 -Year-old $(n=37)$ & 95-Year-old $(n=23)$ & 100 -Year-old $(n=27)$ \\
\hline $\begin{array}{l}\text { Age } \\
\qquad \text { Years, mean } \pm S D\end{array}$ & $85.2 \pm 14.2$ & $60.7 \pm 0.7$ & $81.3 \pm 2.6$ & $91.2 \pm 1.5$ & $95.9 \pm 1.1$ & $100.9 \pm 1.2$ \\
\hline $\begin{array}{l}\text { Neopterin } \\
\quad n M, \text { median }(I Q R)\end{array}$ & $\begin{array}{l}20.3 \\
(11.7-32.2)\end{array}$ & $\begin{array}{l}10.4 \\
(5.3-18.0)\end{array}$ & $\begin{array}{l}18.9 \\
(13.8-32.2)\end{array}$ & $\begin{array}{l}22.5 \\
(15.0-34.1)\end{array}$ & $\begin{array}{l}27.6 \\
(22.3-36.7)\end{array}$ & $\begin{array}{l}19.1 \\
(12.9-26.0)\end{array}$ \\
\hline $\begin{array}{l}\text { Creatinine } \\
\qquad m g / d l, \text { median }(I Q R)\end{array}$ & $\begin{array}{l}0.9 \\
(0.8-1.2)\end{array}$ & $\begin{array}{l}0.8 \\
(0.7-0.9)\end{array}$ & $\begin{array}{l}0.8 \\
(0.7-1.2)\end{array}$ & $\begin{array}{l}1.0 \\
(0.7-1.3)\end{array}$ & $\begin{array}{l}1.1 \\
(0.9-1.3)\end{array}$ & $\begin{array}{l}1.0 \\
(0.8-1.3)\end{array}$ \\
\hline $\begin{array}{l}\text { Glutathione } \\
\qquad \mu M \text {, median }(I Q R)\end{array}$ & $\begin{array}{l}3.9 \\
(2.7-5.4)\end{array}$ & $\begin{array}{l}3.4 \\
(2.0-4.3)\end{array}$ & $\begin{array}{l}3.3 \\
(2.5-5.0)\end{array}$ & $\begin{array}{l}4.0 \\
(2.7-6.4)\end{array}$ & $\begin{array}{l}4.3 \\
(3.1-5.3)\end{array}$ & $\begin{array}{l}4.5 \\
(3.3-6.2)\end{array}$ \\
\hline $\begin{array}{l}\text { Folate } \\
\qquad n M, \text { median }(I Q R)\end{array}$ & $\begin{array}{l}5.6 \\
(4.2-7.5)\end{array}$ & $\begin{array}{l}6.8 \\
(5.9-8.8)\end{array}$ & $\begin{array}{l}6.8 \\
(4.9-10.1)\end{array}$ & $\begin{array}{l}4.5 \\
(4.1-6.0)\end{array}$ & $\begin{array}{l}4.3 \\
(3.2-6.1)\end{array}$ & $\begin{array}{l}4.3 \\
(2.5-6.7)\end{array}$ \\
\hline $\begin{array}{l}\text { Vitamin B12 } \\
\quad p g / m L, \text { median }(I Q R)\end{array}$ & $\begin{array}{l}373 \\
(286-523)\end{array}$ & $\begin{array}{l}480 \\
(342-584)\end{array}$ & $\begin{array}{l}341 \\
(276-399)\end{array}$ & $\begin{array}{l}343 \\
(253-396)\end{array}$ & $\begin{array}{l}426 \\
(307-507)\end{array}$ & $\begin{array}{l}314 \\
(236-506)\end{array}$ \\
\hline
\end{tabular}

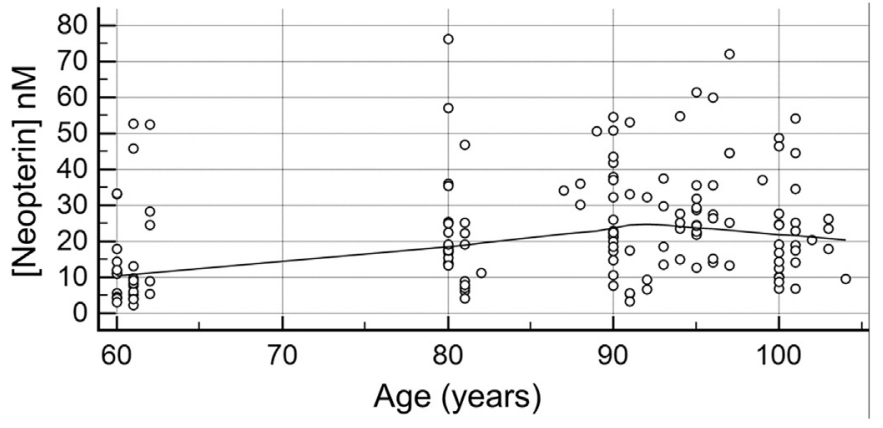

Fig. 1. Graphical relationship between neopterin concentrations and age with trend line computed by local regression smoothing method. Spearman's coefficient of rank correlation was 0.27 and it was significant at $P=0.0008$.

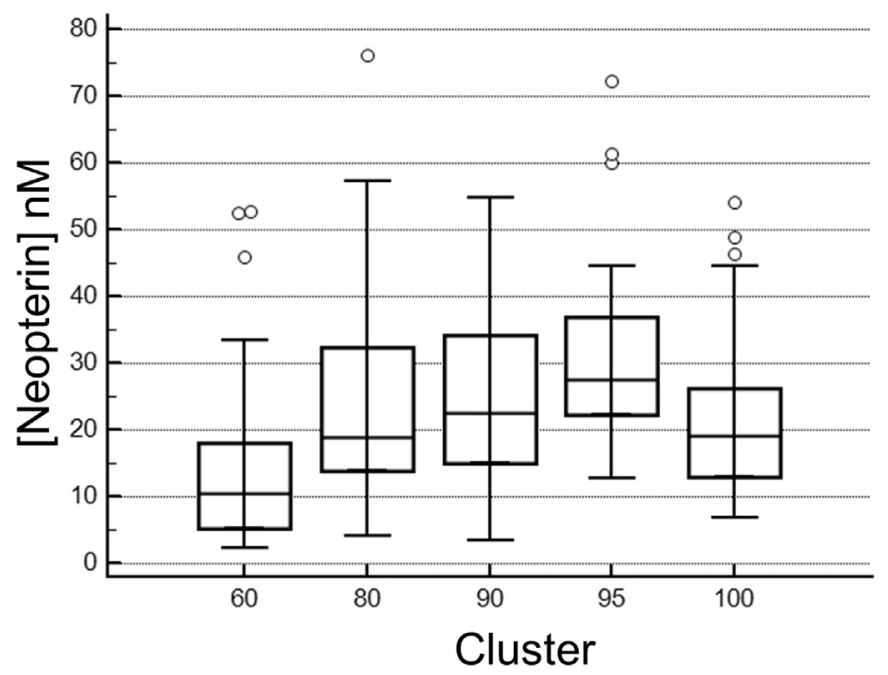

Fig. 2. Pairwise comparisons of subgroups according to age clusters. 60-year-old group was statistically different from 80-, 90-, 95-, and 100-year-old groups. 80-year-old group was statistically different from 60-, and 95-year-old groups. 90-year-old group was statistically different from 60-year-old group. 95-year-old group was statistically different from 60-, 80-, and 100-year-old groups. 100-year-old group was statistically different from 60- and 95-year-old groups. Central box in the Whisker plot represents the values from the lower to upper quartile ( 25 to 75 percentile) while the middle line is the median value. The lines departing from the lower and upper quartiles indicate the minimum and maximum value, excluding outside (inner fences) and far out (outer fences) values.

(95.9 \pm 1.1 years). However, in the 100-year-old group neopterin concentrations were significantly lower than those of the 95-year-old group. The trend to lower neopterin concentrations in the 100-year-old cluster vs. the 95-year-old group, assessed by Jonckheere-Terpstra trend test, was significant at $P=0.024$ (Fig. 3). The median creatinine concentration in the whole study population was $0.9(0.8-1.2) \mathrm{mg} / \mathrm{dL}$, and

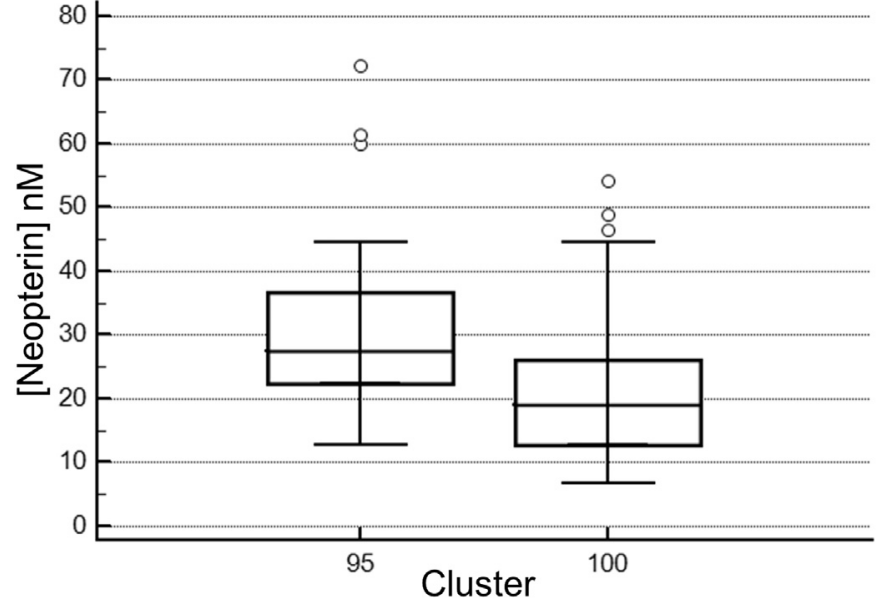

Fig. 3. Trend to lower neopterin concentrations in the 100 -year-old cluster vs. the 95 year-old group was significant at $P=0.024$ (Jonckheere-Terpstra trend test).

there was a significant gender-related difference (females 0.8 (IQR $0.7-1.0) \mathrm{mg} / \mathrm{dL}$ vs. males 1.1 (IQR $0.9-1.5$ ) $\mathrm{mg} / \mathrm{dL}, P<0.0001$ ). Except for the 60-year-old group no significant difference in creatinine concentrations among the other age groups was observed. The median GSH concentration in the whole study group was 3.9 (IQR 2.7-5.4) $\mu \mathrm{M}$, and there were no significant gender-related differences (females 4.0 (IQR 2.8-5.6) $\mu \mathrm{M}$ vs. males 3.6 (IQR 2.4-5.1) $\mu \mathrm{M}, P=0.25$ ). GSH concentrations showed a significant trend to increase with advancing age, with the centenarians showing the highest concentrations. However, no significant differences between GSH concentrations were observed among the 90-, 95-, and 100-year-old groups. Median values of serum folic acid in the whole study population were 5.6 (IQR 4.2-7.5) $\mathrm{nM}$, and they were significantly higher in females than males (females 6.0 (IQR 4.3-8.3) nM vs. males 4.8 (IQR 4.0-6.5) $\mathrm{nM}, P=0.04$ ). There was no significant difference in serum folic acid concentrations among the 90-, 95-, and 100-year-old groups. Median concentrations of vitamin B12 were 373 (IQR 286-481) pg/mL, and they did not differ between males and females (females 395 (IQR 288-544) pg/mL vs. males 335 (IQR 277-429) pg/mL, $P=0.25$ ). Moreover, no difference between the five age groups was observed. Considering the neopterin mean values for the 95-years old group and centenarians observed in our study $(31.8 \pm 15.8 \mathrm{nM}$ vs. $22.5 \pm 13.1 \mathrm{nM})$ and a type I error alpha of 0.05 , a post-hoc sample size to have a power of $80 \%, 90 \%$, $99.95 \%$, and $99.99 \%$ was, respectively, $18,23,28$, and 39 subjects. In our study, the sample size in the 95-years old group and the centenarians was, respectively, 23 and 27 subjects, which allows for a power of at least $90 \%$. 


\section{Discussion}

The measurement of neopterin in body fluids allows to sensitively monitor the degree of immune activation (Fuchs et al., 1992) and, therefore, of the immune processes that appear to underlie key agerelated diseases affecting life expectancy in older adults. In this analysis of Sardinian older adults, the whole group of participants (mean age $85.25 \pm 14.20$ years) had a median neopterin value of 20.35 (IQR 11.66-32.23) nM. Although slightly greater than the average levels reported by other authors, these values are consistent with the upper limits of normal reported by Reibnegger et al. (1988) (19.0 nM above 75 years). As reported in earlier studies (Diamondstone et al., 1994; Schennach et al., 2002), we also found that plasma neopterin concentrations significantly correlate with age, suggesting increased monocyte/macrophage-mediated immune activation with advancing age. Indeed, there is good evidence that the immune system in aging acts in a proinflammatory fashion (Franceschi et al., 2000) and, in this sense, increased concentrations of proinflammatory markers including neopterin have been well documented in healthy older people (Catania et al., 1997; Myśliwska et al., 1998; Murr et al., 2004). On the other hand, a proinflammatory environment might partially compensate for the declining T-cell function detected in old age (Baggio et al., 1998; Franceschi et al., 2000). The five age clusters selected for this study showed significantly different values for plasma neopterin and, as already observed by Aulitzky et al. (1988), there was no statistically significant sex-related difference. As expected for healthy subjects, the 60-year-old group had the lower neopterin levels, followed by 80-, 90-, and 95-year-old groups. This was compatible with their stated health status, as about $60 \%$ of the individuals of the latter groups received a physician diagnosis of at least one chronic medical condition. It should be noted, in addition, that the clinical status of the subjects might be even underestimated as many geriatric conditions, including common ones such as sarcopenia and asthma are often underrecognized by physicians (Fielding et al., 2011; Gillman and Douglass, 2012). On the other hand, it is also possible that in some apparently healthy subjects, the pathological process may have already started while being clinically latent. Notably, the group of centenarians, despite a clinical status comparable to that of other age groups, had neopterin values comparable to those of 80 - and 90-year-old groups, and significantly lower than that of 95-year-old group. The similarities in the prevalence of chronic disease burden between the centenarians and the other study groups do not explain the reported difference in neopterin concentrations. The latter are also unlikely to be secondary to changes in renal function, as described by other authors (Lhee et al., 2006). Although significantly lower in the 60-year-old group and despite it does not accurately reflect changes in renal function in older people as muscle mass and dietary protein intake affect its levels, serum creatinine concentrations, in fact, did not differ significantly among the 80-, 90-, 95-, and 100-year-old groups. On these bases, therefore, a different nutritional state among of the individuals in these age groups can be also excluded and, in this sense, markers of nutritional state, i.e. serum folic acid and vitamin B12 concentrations, were not dissimilar in the 90, 95-, and 100-year-old groups. From an immunity point of view, therefore, this finding reveals that centenarians seem younger than their age, in accordance with earlier observations reporting that immune responses in healthy centenarians were closer to those observed in younger adults than those in 70-year-old subjects (Alonso-Fernandez et al., 2008; De la Fuente, 2008). The lower neopterin concentrations in centenarians, therefore, could suggest that a reduced activation of the aged immune system might be associated with improved survival and health status. This is consistent with the observations that the elevation of neopterin concentrations are related to increased frailty (Leng et al., 2011) and decreased humoral immunity in older adults (Murr et al., 2003) as well as to shorter residual life span of the nonagenarians (Solichova et al., 2001). Furthermore, higher neopterin production is also associated with an exhausted pool of antioxidants (Murr et al., 2009) and increased oxidation products such as lipid peroxidation, oxidized proteins, or DNA. Moreover, neopterin was found itself to enhance the toxic effects induced by reactive oxygen species (Murr et al., 1999). Theoretically, the smaller values of neopterin in centenarians, along with the age-associated trend towards higher GSH concentrations, could, therefore, suggest both a reduced oxidative metabolism and a lower oxidative damage of macromolecules because of the oxidative stress emerging during the cell-mediated immune response. This was consistent with the previous observations that extreme longevity is associated with a lower degree of oxidative stress than that experienced by middle-aged adults (Paolisso et al., 1998; Barbieri et al., 2003; Alonso-Fernandez et al., 2008).

\section{Conclusions}

In this study, we observed in Sardinian centenarians a significant inversion of the age-associated trend to increasing neopterin concentrations. This finding would suggest that plasma neopterin concentrations might be a useful predictor of survival and health status. The lower neopterin concentrations in centenarians, in fact, are consistent with the view of a reduced inflammation and oxidative stress status in these subjects when compared with other subgroups within the old-old population. However, as our study shows limitations including the relatively small sample size, its cross-sectional design, and the lack of other measures of inflammation and oxidative stress status in the observed population, further studies that include prospective investigations are needed to understand the biological processes that underlie this apparently preserved immune status of centenarians and its impact on the longevity.

\section{Acknowledgments}

This study was funded by Ministero dell'Istruzione, dell'Università e della Ricerca (MIUR, Italy) - Progetti di Ricerca di Rilevante Interesse Nazionale - PRIN 2015 (Prot. 20157ATSLF_002).

Professor Arduino A. Mangoni contributed to this study during a Visiting Professorship at the University of Sassari.

\section{References}

Alonso-Fernandez, P., Puerto, M., Mate, I., Ribera, J.M., de la Fuente, M., 2008. Neutrophils of centenarians show function levels similar to those of young adults. J. Am. Geriatr. Soc. 56, 2244-2251.

Aulitzky, W.E., Tilg, H., Niederwieser, D., Riccabona, G., Obendorf, L., Margreiter, R., Pfaller, W., Huber, C., 1988. Comparison of serum neopterin levels and urinary neopterin excretion in renal allograft recipients. Clin. Nephrol. 29, 248-252.

Baggio, G., Donazzan, S., Monti, D., Mari, D., Martini, S., Gabelli, C., Dalla Vestra, M., Previato, L., Guido, M., Pigozzo, S., Cortella, I., Crepaldi, G., Franceschi, C., 1998. Lipoprotein(a) and lipoprotein profile in healthy centenarians: a reappraisal of vascular risk factors. FASEB J. 12, 433-437.

Barbieri, M., Rizzo, M.R., Manzella, D., Grella, R., Ragno, E., Carbonella, M., Abbatecola, A.M., Paolisso, G.1., 2003. Glucose regulation and oxidative stress in healthy centenarians. Exp. Gerontol. 38, 137-143.

Baylis, D., Bartlett, D.B., Patel, H.P., Roberts, H.C., 2013. Understanding how we age: insights into inflammaging. Longev Healthspan 2, 8.

Capuron, L., Geisler, S., Kurz, K., Leblhuber, F., Sperner-Unterweger, B., Fuchs, D., 2014. Activated immune system and inflammation in healthy ageing: relevance for tryptophan and neopterin metabolism. Curr. Pharm. Des. 20, 6048-6057.

Carru, C., Zinellu, A., Sotgia, S., Serra, R., Usai, M.F., Pintus, G.F., Pes, G.M., Deiana, L., 2004. A new HPLC method for serum neopterin measurement and relationships with plasma thiols levels in healthy subjects. Biomed. Chromatogr. 18, 360-366.

Catania, A., Airaghi, L., Motta, P., Manfredi, M.G., Annoni, G., Pettenati, C., Brambilla, F., Lipton, J.M., 1997. Cytokine antagonists in aged subjects and their relation with cellular immunity. J. Gerontol. A Biol. Sci. Med. Sci. 52, B93-97.

Conover, W.J., 1999. Practical Nonparametric Statistics, 3rd edition. John Wiley \& Sons, New York.

De la Fuente, M., 2008. Role of neuroimmunomodulation in aging. Neuroimmunomodulation 15, 213-223.

Diamondstone, L.S., Tollerud, D.J., Fuchs, D., Wächter, H., Brown, L.M., Maloney, E., Kurman, C.C., Nelson, D.L., Blattner, W.A., 1994. Factors intluencing serum neopterin and $\beta 2$-microglobulin levels in healthy diverse population. J. Clin. Immunol. 14, $368-374$.

Fielding, R.A., Vellas, B., Evans, W.J., Bhasin, S., Morley, J.E., Newman, A.B., Abellan, 
van Kan G., Andrieu, S., Bauer, J., Breuille, D., Cederholm, T, Chandler, J., De Meynard, C., Donini, L., Harris, T., Kannt, A., Keime Guibert, F., Onder, G., Papanicolaou, D., Rolland, Y., Rooks, D., Sieber, C., Souhami, E., Verlaan, S., Zamboni, M., 2011. Sarcopenia: an undiagnosed condition in older adults. Current consensus definition: prevalence, etiology, and consequences. J. Am. Med. Dir. Assoc 12, 249-256.

Franceschi, C., Campisi, J., 2014. Chronic inflammation (inflammaging) and its potential contribution to age-associated diseases. J. Gerontol. Ser. A. 69, S4-S9.

Franceschi, C., Bonafè, M., Valensin, S., Olivieri, F., De Luca, M., Ottaviani, E., De Benedictis, G., 2000. Inflamm-aging. An evolutionary perspective on immunosenescence. Ann. N. Y. Acad. Sci. 908, 244-254.

Fuchs, D., Weiss, G., Reibnegger, G., Wachter, H., 1992. The role of neopterin as a monitor of cellular immune activation in transplantation, inflammatory, infectious, and malignant diseases. Crit. Rev. Clin. Lab. Sci. 29, 307-341.

Fulop, T., Dupuis, G., Baehl, S., Le Page, A., Bourgade, K., Frost, E., Witkowski, J.M., Pawelec, G., Larbi, A., Cunnane, S., 2016. From inflamm-aging to immune-paralysis: a slippery slope during aging for immune-adaptation. Biogerontology 17, 147-157.

Gillman, A., Douglass, J.A., 2012. Asthma in the elderly. Asia Pac. Allergy 2, 101-108.

Ginaldi, L., Loreto, M.F., Corsi, M.P., Modesti, M., De Martinis, M., 2001. Immunosenescence and infectious diseases. Microbes Infect. 3, 851-857.

Green, R., 2011. Indicators for assessing folate and vitamin B-12 status and for monitoring the efficacy of intervention strategies. Am. J. Clin. Nutr. 94, 666S-6672S.

Hamerlinck, F.F., 1999. Neopterin: a review. Exp. Dermatol. 8, 167-176.

Leng, S.X., Tian, X., Matteini, A., Li, H., Hughes, J., Jain, A., Walston, J.D., Fedarko, N.S., 2011. IL-6-independent association of elevated serum neopterin levels with prevalent frailty in community-dwelling older adults. Age Ageing 40, 475-481.

Lhee, H.Y., Kim, H., Joo, K.J., Jung, S.S., Lee, K.B., 2006. The clinical significance of serum and urinary neopterin levels in several renal diseases. J. Korean Med. Sci. 21, 678-682.

Licastro, F., Grimaldi, L.M., Bonafe, M., Martina, C., Olivieri, F., Cavallone, L. Giovanietti, S., Masliah, E., Franceschi, C., 2003. Interleukin-6 gene alleles affect the risk of Alzheimer's disease and levels of the cytokine in blood and brain. Neurobiol. Aging 24, 921-926.

Melichar, B., Solichová, D., Freedman, R.S., 2006. Neopterin as an indicator of immune activation and prognosis in patients with gynecological malignancies. Int. J. Gynecol. Cancer 16, 240-252.

Murr, C., Fuith, L.C., Widner, B., Wirleitner, B., Baier-Bitterlich, G., Fuchs, D., 1999. Increased neopterin concentrations in patients with cancer: indicator of oxidative stress? Anticancer Res. 19, 1721-1728.

Murr, C., Widner, B., Wirleitner, B., Fuchs, D., 2002. Neopterin as a marker for immune system activation. Curr. Drug Metab. 3, 175-187.

Murr, C., Hainz, U., Asch, E., Berger, P., Jenewein, B., Saurwein-Teissl, M., GrubeckLoebenstein, B., Fuchs, D., 2003. Association of increased neopterin production with decreased humoral immunity in the elderly. Exp. Gerontol. 38, 583-587.

Murr, C., et al., 2004. Increased neopterin concentration in older age coincides with decline of CD28 + CD45RA + T-cells. Pteridines 15, 170-174.

Murr, C., Winklhofer-Roob, B.M., Schroecksnadel, K., Maritschnegg, M., Mangge, H., Böhm, B.O., Winkelmann, B.R., März, W., Fuchs, D., 2009. Inverse association between serum concentrations of neopterin and antioxidants in patients with and without angiographic coronary artery disease. Atherosclerosis 202, 543-549.

Myśliwska, J., Bryl, E., Foerster, J., Myśliwski, A., 1998. Increase of interleukin 6 and decrease of interleukin 2 production during the ageing process are influenced by the health status. Mech. Ageing Dev. 100, 313-328.

Oishi, Y., Manabe, I., 2016. Macrophages in age-related chronic inflammatory diseases. NPJ Aging Mech. Dis. 2, 16018-16025.

Paolisso, G., Tagliamonte, M.R., Rizzo, M.R., Manzella, D., Gambardella, A., Varricchio, M., 1998. Oxidative stress and advancing age: results in healthy centenarians. J. Am. Geriatr. Soc. 46, 833-838.

Passarino, G., Calignano, C., Vallone, A., Franceschi, C., Jeune, B., Robine, J.M., Yashin, A.I., Cavalli Sforza, L.L., De Benedictis, G., 2002. Male/female ratio in centenarians: a possible role played by population genetic structure. Exp. Gerontol. 37, 1283-1289.

Reibnegger, G., Huber, L.A., Jürgens, G., Schönitzer, D., Werner, E.R., Wachter, H., Wick, G., Traill, K.N., 1988. Approach to define 'normal aging' in man, immune function, serum lipids, lipoproteins and neopterin levels. Mech. Ageing Dev. 46, 67-82.

Ridker, P.M., Rifai, N., Stampfer, M.J., Hennekens, C.H., 2000. Plasma concentration of interleukin-6 and the risk of future myocardial infarction among apparently healthy men. Circulation 101, 1767-1772.

Schennach, H., Murr, C., Gächter, E., Mayersbach, P., Schönitzer, D., Fuchs, D., 2002. Factors influencing serum neopterin concentrations in a population of blood donors. Clin. Chem. 48, 643-645.

Shaw, A.C., Goldstein, D.R., Montgomery, R.R., 2013. Age-dependent dysregulation of innate immunity. Nat. Rev. Immunol. 13, 875-887.

Solichova, D., Melichar, B., Blaha, V., Klejna, M., Vavrova, J., Palicka, V., Zadak, Z., 2001. Biochemical profile and survival in nonagenarians. Clin. Biochem. 34, 563-569.

Straub, R., 2015. The Origin of Chronic Inflammatory Systemic Diseases and Their Sequelae.

Wu, D., Meydani, S.N., 2008. Age-associated changes in immune and inflammatory responses: impact of vitamin E intervention. J. Leukoc. Biol. 84, 900-914.

Zinellu, A., Carru, C., Galistu, F., Usai, M.F., Pes, G.M., Baggio, G., Federici, G., Deiana, L., 2003. N-methyl-D-glucamine improves the laser-induced fluorescence capillary electrophoresis performance in the total plasma thiols measurement. Electrophoresis 24, 2796-2804.

Zinellu, A., Carru, C., Usai, M.F., Sotgia, S., Deiana, L., 2004. Determination of creatinine in human serum by short-end injection capillary zone electrophoresis. Electrophoresis 25, 1096-1101. 